\title{
Resection of Sentinel Lymph Nodes by an Extraperitoneal Minilaparoscopic Approach Using Indocyanine Green for Uterine Malignancies: A Preclinical Comparative Study
}

Surgical Innovation

I-7

(C) The Author(s) 2015

Reprints and permissions:

sagepub.com/journalsPermissions.nav DOI: $10.1177 / 15533506 / 5620302$

sri.sagepub.com

(S)AGE

\author{
Hélder Ferreira, $M D^{1,2,3}$, Cristina Nogueira-Silva, $M D, P^{1,2,4}$ \\ Alice Miranda, DVM ${ }^{1,2}$, and Jorge Correia-Pinto, $M D, P^{1,2,4}$
}

\begin{abstract}
Background. The sentinel lymph node (SLN) concept might minimize surgical aggressiveness in cervical and endometrial malignancies. The aim of the study was to test the feasibility and reliability of minilaparoscopic extraperitoneal SLN excision after indocyanine green (ICG) cervical injection using a high-definition near infrared (NIR) imaging system in an in vivo porcine model. The same procedure was performed using conventional laparoscopic instruments and both outcomes were compared. Methods. Twenty-four animals were equally and randomly divided into a minilaparoscopic group (group A) and a 5-mm conventional laparoscopic group (group B). A high-definition NIR imaging system and a $30^{\circ}$ ICG endoscope were used. First, ICG $(0.5 \mathrm{~mL})$ was injected in the paracervical region. The SLN coloring time was recorded. An extraperitoneal approach to the SLN was executed with the same $\mathrm{CO}_{2}$ retropneumoperitoneum pressures $(10 \mathrm{~mm} \mathrm{Hg})$. In both groups, the times for SLN localization and excision, as well as complications, were registered. Finally, a laparotomy was then done to evaluate whether any stained SLN still remained. The same surgical team performed all experiments. Results. SLNs were identified and extraperitoneally excised in all animals without major complications. The SLN localization varied between animals from external iliac to preaortic regions. The surgical times were shorter with minilaparoscopy $(39.3 \pm 13$ minutes) than with conventional 5 -mm instruments $(5 \mid .3 \pm$ I4.17 minutes; $P=.042$ ). In group $B$, one stained SLN remained and was only detected by laparotomy. Conclusions. We confirmed the feasibility and reliability of extraperitoneal minilaparoscopic approach for identification, dissection, and excision of SLN using an NIR imaging system and ICG.
\end{abstract}

\section{Keywords}

gynecologic laparoscopy, surgical oncology, evidence-based medicine/surgery

\section{Introduction}

The evaluation of lymphatic metastases in the staging of cervical and endometrial cancers continues to be controversial in gynecologic oncology. By convention, the entire lymphatic basin draining a tumor is excised (resulting in prolonged operation duration, increased blood loss, infection, nerve injury, lymphocyst formation, vascular injury, venous thromboembolism, and lower extremity lymphedema) to obtain histological diagnosis of lymphatic metastases. ${ }^{1,2}$ The development of sentinel lymph node (SLN) biopsy techniques have reduced the number of complications compared to traditional lymphadenectomy. SLN improves detection of micrometastatic disease and fine-tunes our lymphadenectomy anatomic templates. ${ }^{3-7}$
The gold standard assessment of SLN in cervical and endometrial cancers is still to be established. SLN assessment has been performed with laparotomy, conventional laparoscopy, ${ }^{8}$ robotics, ${ }^{9}$ and minilaparoscopy, ${ }^{10}$ always via transperitoneal or intraperitoneal approach.

\footnotetext{
'University of Minho, Braga, Portugal

${ }^{2}$ ICVS/3B's—PT Government Associate Laboratory, Braga, Portugal

${ }^{3}$ Centro Hospitalar do Porto, Porto, Portugal

${ }^{4}$ Hospital de Braga, Braga, Portugal

Corresponding Author:

Hélder Ferreira, Rua Júlio Dinis, No. 230, B-2, $9^{\circ}$ Esq, 4050-3। 8

Porto, Portugal.

Email: helferreira@hotmail.com
} 
The benefits conferred by an extraperitoneal, laparoscopic lymphadenectomy include a short learning curve, decreased adhesion formation, feasibility in obese patients, patients with peritoneal adhesions, and reduced bowel complications associated with postoperative adjuvant irradiation. ${ }^{11}$

To the authors' knowledge, the combination of extraperitoneal minilaparoscopy in SLN assessment has not previously been described. There are no data referring to the use of these smaller instruments in the reduced extraperitoneal space. The aim of the study was to test the feasibility and reliability of minilaparoscopic extraperitoneal SLN excision after indocyanine green (ICG) cervical injection using a high-definition near infrared (NIR) imaging system in an in vivo porcine model. The same procedure was performed using 5-mm conventional laparoscopic instruments and both outcomes were compared.

\section{Materials and Methods}

Animal experiments were performed following EU Directive 2010/63/EU and the Portuguese law for animal welfare (Diário da República, Portaria 1005/92).

\section{Animal Model}

Our cohort included 27 female pigs (Sus scrofus domesticus) weighing between 35 and $45 \mathrm{~kg}$. The first 3 pigs were used to establish anatomical landmarks and technical steps and therefore were not included in the subsequent protocol. Twenty-four pigs were equally and randomly divided into a minilaparoscopic group (group A) and a conventional laparoscopic group (group B). The mean weight of the pigs was $37 \pm 2.1 \mathrm{~kg}$ in group A and $37 \pm 1.6 \mathrm{~kg}$ in group B. All pigs were 12 weeks of age.

\section{Pig Preparation}

All procedures were performed with the pigs under general anesthesia, with endotracheal intubation and mechanical ventilation. The pigs were preoperatively starved of food for 8 hours and water for 4 hours. Animals were premedicated with a combination of azaperone $(4 \mathrm{mg} / \mathrm{kg}$, intramuscularly [IM]), midazolam ( $1 \mathrm{mg} / \mathrm{kg}$, IM), and atropine $(0.05 \mathrm{mg} / \mathrm{kg}$, IM). The pigs were initially anaesthetized with intravenous propofol $(6 \mathrm{mg} / \mathrm{kg})$. Anesthesia was maintained with continuous intravenous propofol infusion $(20 \mathrm{mg} / \mathrm{kg} / \mathrm{h})$ and intramuscular buprenorphine $(0.05 \mathrm{mg} / \mathrm{kg})$.

\section{Surgical Procedures}

After anaesthetization of the pigs, the pigs were positioned in a dorsal Trendelenburg position and immobilized. In both groups, pneumoperitoneum was created using a Veress needle.

A special laparoscope (26003 BGA, Karl Storz GmbH $\&$ Co KG, Tuttlingen, Germany) with a $30^{\circ}$ angle of view, incorporating observation filters that allowed NIR and white light imaging without changing the telescope. The powerful xenon light source (D-LIGHT P SCB, Karl Storz) provided both visible and NIR excitation light. A pedal controlled by the surgeon was used to switch from standard light to NIR. The use of a fluid light cable ensured high light transmission. A dedicated, infraredsensitive camera head (IMAGE 1 H3-Z FI, Karl Storz) in conjunction with a full high-definition camera control unit (IMAGE 1, Karl Storz) was used to process the genuine NIR fluorescence.

First, a laparoscope was introduced by a supraumbilical trocar and $0.5 \mathrm{~mL}$ of ICG solution (25 mg of ICG [Pulsion Medical Systems AG, Munchen, Germany] diluted in $10 \mathrm{~mL}$ of $0.9 \% \mathrm{NaCl}$ solution) was injected on the paracervical region using a long needle (Spinocan 26G 120 mm; Braun, Melsungen, Germany). A few minutes after ICG injection, the colored lymphatic channels were mapped and followed toward nodes that retained the ICG dye. A stopwatch was used to calculate the time required for coloring of the nodes.

Second, a 12-mm incision, $2 \mathrm{~cm}$ superomedial to the anterosuperior iliac spine was made to develop an extraperitoneal space. Once the peritoneal layer was visualized, the surgeon introduced one finger into the incision and performed blunt dissection to separate the peritoneal layer from the abdominal wall, which was constantly visualized via the umbilical port. Subsequently, a 10-mm, single use, blunt tip trocar (OMST10BT, AutoSuture, Covidien) was placed through the incision to develop an extraperitoneal space. The abdomen was deflated, and the laparoscope was brought to the balloon-tipped trocar in the extraperitoneal space, which was then insufflated. The retroperitoneum was insufflated with carbon dioxide $\left(\mathrm{CO}_{2}\right)$ with a pressure not exceeding $10 \mathrm{~mm} \mathrm{Hg}$. The loose, areolar tissue in the extraperitoneal space was dissected freely using the laparoscope. Special attention was taken to prevent perforating the peritoneum, which could compromise the retropneumoperitoneum.

In the minilaparoscopic approach (group A), a 3.5-mm trocar was introduced into the extraperitoneal space along the midaxillary line, under the subcostal margin, approximately $5 \mathrm{~cm}$ above the initial trocar. An additional 3.5$\mathrm{mm}$ trocar was then placed 3 to $4 \mathrm{~cm}$ cephalad to the balloon-tipped trocar in the preperitoneal space under endoscopic guidance. Utmost care was taken to ensure accurate trocar placement without perforating the peritoneum, which would make the remaining procedure difficult. While advancing to the retroperitoneum, $\mathrm{CO}_{2}$ was administered via the balloon trocar channel to keep good 


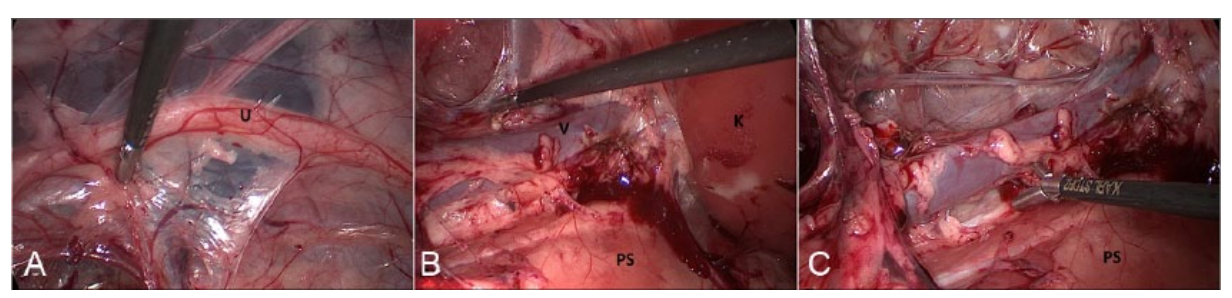

Figure I. Extraperitoneal view (U, ureter; V, vena cava; PS, psoas muscle; K, kidney).

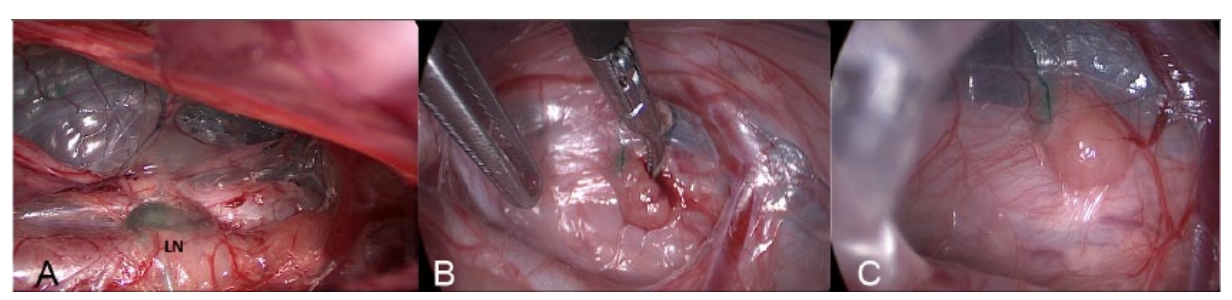

Figure 2. Lymphatics and lymph nodes (LN) colored by ICG.

exposure. The instrumental trocars accommodated 36-cm-long minilaparoscopic instruments (Karl Storz Endoskope minilaparoscopy instruments set), including graspers, cold scissors, suction/irrigation, and the recent 3.5-mm bipolar coagulator (Karl Storz Robi).

Once the extraperitoneal space was adequately insufflated, the left psoas muscle, ureter, gonadal vessels, and common iliac artery were easily identified (Figure 1). The retroperitoneum was accessed with care to avoid bleeding from vessels and capillaries, which could stain the retroperitoneum, making it difficult to follow the colored lymphatic channels. The lumbar and iliac segments of the ureter were lifted along with the peritoneal sac. The lateral aspect of the iliac artery was used as a guide to dissect caudally to the level of its bifurcation, up to the aortic bifurcation and lastly to the renal vessels. The operative field was thoroughly evaluated to look for colored retroperitoneal lymph nodes (Figure 2). The ureters and promontory were 2 useful landmarks in following the path of the lumboaortic lymph nodes. At the level of the kidneys, we identified paracaval, aortocaval, and preaortic lymph nodes. The laparoscopic fluorescence imaging system could visualize the lymphatic channels and nodes by stepping on the pedal (Figure 3 ). The distance between the laparoscope tip and target when applying the fluorescence was standardized at 2 to $3 \mathrm{~cm}$. Once a colored lymph node was confirmed, it was grasped and gently separated from the attached lymphatic tissue (Figure 4). The peritoneal sac was elevated from the left common iliac vein and then from the sacral promontory. The bifurcation of the inferior vena cava was identified. The right

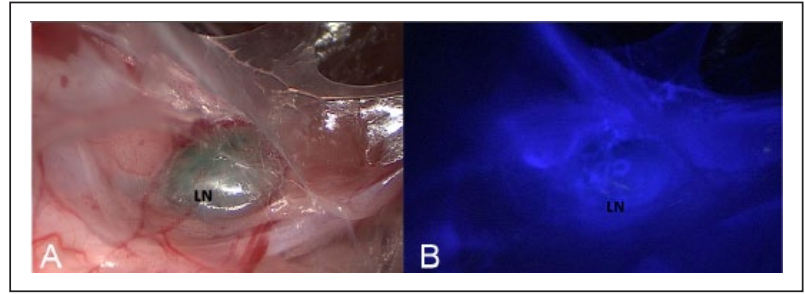

Figure 3. (A) Lymph node colored by ICG. (B) Lymph node in NIR light.

common iliac vein followed by the right common iliac artery were freed using blunt dissection. The right ureter was then elevated and separated from the iliac vessels and the psoas muscle. At this time, the right lateral common iliac, presacral, and precaval nodes were identified. Lymphatic vessels were sealed with the bipolar dissector (Figure 5). The resected SLNs were extracted from the extraperitoneal cavity with an endoscopic bag through the $10-\mathrm{mm}$ port under direct vision of a 3-mm endoscope inserted at one of the $3.5-\mathrm{mm}$ ports. Careful hemostasis with bipolar energy was used at the end of the procedure. At this time, the extraperitoneal space was deflated, and the abdominal cavity was insufflated. The laparoscope was once again placed through the supraumbilical port. Finally, a laparotomy was then performed to evaluate whether there were any remaining SLNs. After the procedure, the pigs were euthanized with an overdose of anesthesia. The procedure time (defined as interval between skin incision to SLN removal), difficulties, and complications at each step of the procedure were recorded. The 


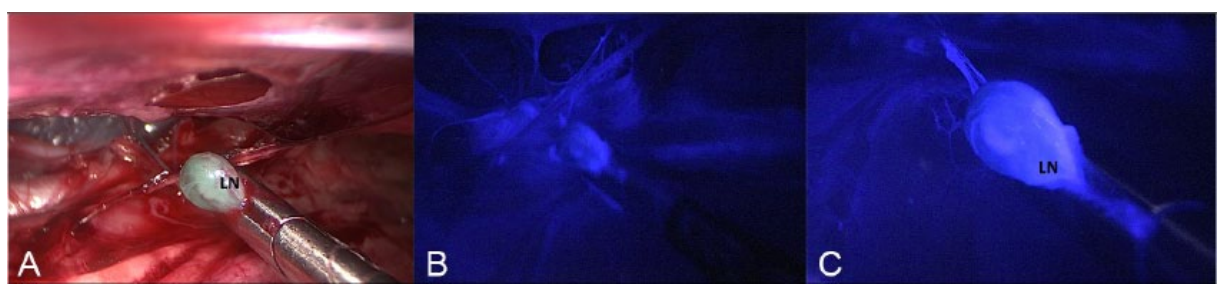

Figure 4. (A) SLN extracted with a minilaparoscopic grasper. (B and C) Lymph node in NIR light.

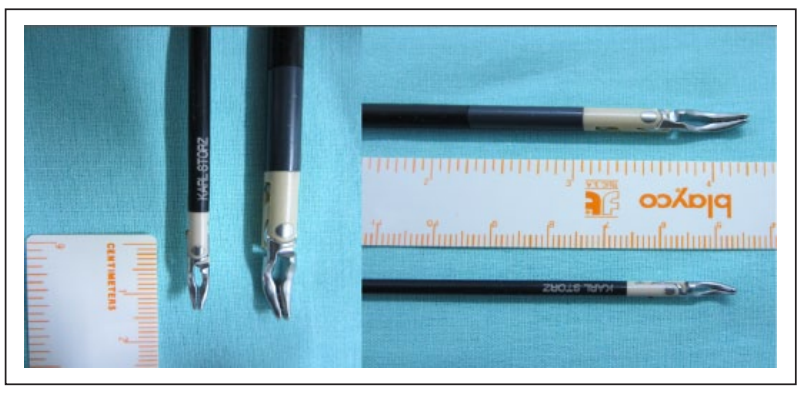

Figure 5. Standard 5-mm rotating bipolar versus minilaparoscopic bipolar dissector.

intraoperative complications were defined as bowel, bladder, ureteral, or vascular injuries, and an estimated blood loss $>200 \mathrm{~mL}$. Estimated blood loss was assessed from the contents of suction devices. The procedure can be broken into 4 parts: diagnostic laparoscopy to observe lymphatic mapping and rule out intraperitoneal disease; the insertion of the retroperitoneal minilaparoscopic trocars; the development of the retroperitoneal space; and the identification, dissection, and resection of SLNs.

In the conventional laparoscopic group (group B), the exact same procedure was performed but with $5-\mathrm{mm}$ conventional laparoscopic instruments. To avoid possible bias between the groups, the same surgical team performed all the experiments.

\section{Histologic Analysis}

The lymph nodes were analyzed without freezing. Each lymph node was fixed in neutral buffered formaldehyde for 72 hours, cut into 0.1-cm-thick slices, and embedded in a paraffin block. Multiple sections were prepared from each block. A set of 3- to 4- $\mu$ m-thick sections was cut every $250 \mu \mathrm{m}$. Sections were stained with hematoxylin and eosin. The images were taken with an Olympus microscope BX61 (Olympus, Tokyo, Japan).

\section{Statistical Analysis}

All quantitative data are presented as mean \pm SD. Statistical analysis was performed using the statistical software SigmaStat (version 3.5; Systat Software Inc, San Jose, CA). Independent samples Student $t$-test analysis was used to compare surgical times, and statistical significance was set at $P<.05$. We also present Cohen $d$ measure of effect size, whereby 0.2 equates to a small effect, 0.5 equates to a medium effect, and effects $>0.8$ equates to large effects. ${ }^{12}$

\section{Results}

Following the ICG injection in the paracervical region, the green lymphatic channels were identified, as well as the ending colored lymph nodes, which were considered SLNs. The average time to coloring was $3.58 \pm 1.38 \mathrm{~min}$ utes in group $\mathrm{A}$ and $3.25 \pm 1.42$ minutes in group $\mathrm{B}(t[22]$ $=0.58 ; P=.57, d=0.23$ - small effect size). Extraperitoneal dissection and excision of SLNs with 3-mm instruments were performed with success in all pigs. The overall results of our study are summarized in Table 1.

The average times to perform the experiments were $39.3 \pm 10.7$ minutes in group $\mathrm{A}$ and $51.3 \pm 14.17$ minutes in group $\mathrm{B}(P=.042)$. From experiment 1 to experiment 12 , the operative time was dramatically reduced (from 65 to 25 minutes in group A and from 79 to 34 minutes in group B) presumably due to improved experience and identification of the anatomic landmarks.

In group $\mathrm{A}$, the intraoperative complications included intraoperative bleeding ( 40 and $30 \mathrm{~mL}$ ) in 2 cases, which was controlled by minilaparoscopic instruments, and 1 diffuse anterior abdominal wall emphysema. In group B, there were 2 cases of intraoperative bleeding (60 and 40 $\mathrm{mL}), 1$ case of abdominal wall emphysema, and 2 pneumoperitoneums (one of which was associated with peritoneal perforation and both were evacuated by Veress needle insertion). There were no cases with urinary and digestive tract injuries. During the procedures, the heart rate, arterial pressure, and respiratory function were stable. In group B, there was only SLNs detected after laparotomy.

Microscopic examination of the sentinel nodes removed with minilaparoscopy revealed intact nodes with complete preservation of nodal architecture and no evidence of thermal or mechanical damage. 
Table I. Perioperative Data After ICG Injection and SLN Removal.

\begin{tabular}{|c|c|c|c|c|c|c|}
\hline \multicolumn{3}{|c|}{ Perioperative Variables } & \multirow{2}{*}{$\begin{array}{c}\text { Group A: } \\
\text { Minilaparoscopy }(\mathrm{n}=24) \\
3.58 \pm 1.38(\mathrm{I}-6)\end{array}$} & \multirow{2}{*}{$\begin{array}{l}\text { Group B: Conventional } \\
\text { Laparoscopy }(n=24) \\
3.25 \pm 1.42(1-6)\end{array}$} & \multirow{2}{*}{$\frac{P \text { Value }}{.57}$} & \multirow{2}{*}{$\frac{\text { Cohen's d }}{0.23}$} \\
\hline SLN & Coloring & lutes), mean \pm SD (range) & & & & \\
\hline & Number & mean $\pm \mathrm{SD}$ (range) & $1.9 \pm 0.8(I-3)$ & $2 \pm 0.74(I-3)$ & & \\
\hline & Location & Preaortic & 3 & 6 & & \\
\hline & & Left iliac vessels & 9 & 7 & & \\
\hline & & Right iliac vessels & 5 & 6 & & \\
\hline & & Promontory & 4 & 4 & & \\
\hline & & Right obturator fossa & 1 & - & & \\
\hline & & Left obturator fossa & 1 & 1 & & \\
\hline \multicolumn{3}{|c|}{ Operative time (minutes), mean \pm SD (range) } & $39.3 \pm 13.02(25-65)$ & $51.3 \pm 14.17(34-79)$ & .042 & 0.85 \\
\hline
\end{tabular}

Abbreviations: ICG, indocyanine green; SLN, sentinel lymph node. ${ }^{\text {a}}$ Student's $t$ test.

\section{Discussion}

SLN techniques are now part of the standard treatment for breast cancer ${ }^{13}$ melanoma,${ }^{14}$ and selected cases of vulvar cancer. ${ }^{15,16}$ SLN biopsy has been one of the more recent, important innovations in surgical oncology. It has been established as less invasive and more informative to clinicians. ${ }^{4,5}$ In uterine cancers, SLN biopsy can potentially provide more accurate identification of uterine drainage, primary tumor staging, and may have applications in fertility-sparing surgical procedures. ${ }^{17}$

In our study, the feasibility and reliability of SLN removal by a retroperitoneoscopic approach using minilaparoscopic instrumentation after ICG dye cervical injection were assessed. This was also compared with conventional 5-mm laparoscopic instruments.

The ICG fluorescent dye relies on a fluorometrically capable camera and appears green when excited by light in the NIR range. It has the potential advantage of being readily visible through visceral fat and has a higher detection rate than blue dye in SLNs. ${ }^{18}$ NIR fluorescence imaging using ICG may improve SLN technology in cervical and endometrial cancers by increasing the rate of bilateral SLN mapping without the added cost and inconvenience of technetium. ${ }^{19}$

Dargent and Salvat reported the first use of conventional laparoscopic instruments for extraperitoneal lymphadenectomy in women with gynecologic cancers. ${ }^{20,21}$ Occelli et al found that compared with transperitoneal approach in animal studies, extraperitoneal laparoscopy leads to fewer postoperative adhesions, lowering the risk of postoperative radiation-related complications. ${ }^{11}$ Additionally, as the peritoneal cavity is untouched, complications such as postoperative ileus, intraperitoneal adhesions, and intestinal obstruction are eliminated. ${ }^{22} \mathrm{~A}$ further important advantage of the extraperitoneal route is the protection of the bowel provided by the peritoneal sac. ${ }^{23}$ It is anatomically and surgically more logical as it is carried out on the left-hand side (providing better access to the aortic territory lymph nodes). ${ }^{24}$ Previous abdominal surgery, radiotherapy, and chemotherapy do not complicate the extraperitoneal approach. The low incidence of postoperative complications and minimal hospital stay promote early initiation of adjuvant therapy. Extraperitoneal techniques may reduce the time taken to access the lymphatic channels since adhesiolysis and bowel mobilization is unnecessary. There is decreased risk of electrosurgical bowel injury and there is no risk for unrecognized enterotomy due to traction or dissection. ${ }^{22}$ The ureters is automatically mobilized out of the dissection field by attachment to the peritoneal envelope. Since the peritoneal sac acts as a natural retractor, fewer instruments may be needed. Finally, there is potentially less pain caused by diaphragmatic irritation from $\mathrm{CO}_{2}$ gas. Potential drawbacks may include subcutaneous emphysema with retropneumoperitoneum, possibly leading to pneumothorax via potential spaces and mediastinal anatomic pathways. ${ }^{22,25}$

Minilaparoscopy, using miniaturized instruments, further reduces perioperative morbidity, enhances cosmetic healing, and avoids incisional herniation. Recently, minilaparoscopic surgical techniques have benefited from additional product development with a focus on improving instrument strength. ${ }^{26}$ During these experiments, researchers found thinner instruments to be functional with no increase in complications. The new minilaparoscopic rotating bipolar instrument allows precise tissue dissection and fine coagulation. The use of small-diameter instruments is feasible with low $\mathrm{CO}_{2}$ pressures, thereby reducing possible complications related to pneumoperitoneum. ${ }^{27}$

In our comparative study, the operating time with minilaparoscopic instruments was shorter than with $5-\mathrm{mm}$ conventional instruments $(P=.042)$ without major complications. These smaller instruments may offer the possibility of working better in restricted and narrow spaces like the retroperitoneum with lower $\mathrm{CO}_{2}$ pressures. 
This is the first study that compares minilaparoscopic versus conventional laparoscopic extraperitoneal approach in SLN removal.

The strengths of our study include the controlled setting in which only a single variable (instruments size) was altered. The same team of surgeons performed all procedures. The most important limitation perhaps is its preclinical nature. Further studies with larger samples, lower $\mathrm{CO}_{2}$ pressures, and local anesthesia ${ }^{28}$ should be done. Our findings may help focus future human studies.

\section{Conclusions}

The resection of SLN by a minilaparoscopic extraperitoneal approach after ICG cervical injection was performed successfully with no major complications. In this preclinical study, the operative technique was feasible and reliable. The surgical time with the minilaparoscopic instruments was shorter than with $5-\mathrm{mm}$ conventional instruments $(P=.042)$. This procedure may reduce morbidity during staging of gynecologic malignancies. Further studies are needed to confirm our results.

\section{Author Contributions}

Study concept and design: Hélder Ferreira

Acquisition of data: Hélder Ferreira, Alice Miranda and Cristina Nogueira-Silva

Analysis and interpretation: Hélder Ferreira, Cristina NogueiraSilva, Alice Miranda and Jorge Correia-Pinto

Study supervision: Jorge Correia-Pinto and Cristina NogueiraSilva

\section{Authors' Note}

Animal experiments were performed following EU Directive 2010/63/EU and the Portuguese law for animal welfare (Diário da República, Portaria 1005/92).

\section{Declaration of Conflicting Interests}

The author(s) declared no potential conflicts of interest with respect to the research, authorship, and/or publication of this article.

\section{Funding}

The author(s) disclosed receipt of the following financial support for the research, authorship, and/or publication of this article: This study was supported by the Science \& Technology Foundation (FCT), Portugal—PTDC/SAU-OSM/105578/2008.

\section{References}

1. Levenback C, Coleman RL, Burke TW, et al. Lymphatic mapping and sentinel node identification in patients with cervix cancer undergoing radical hysterectomy and pelvic lymphadenectomy. J Clin Oncol. 2002;20:688-693.
2. Matsuura Y, Kawagoe T, Toki N, Tanaka M, Kashimura M. Long-standing complications after treatment for cancer of the uterine cervix - clinical significance of medical examination at 5 years after treatment. Int $J$ Gynecol Cancer. 2006;16:294-297.

3. Echt ML, Finan MA, Hoffman MS, Kline RC, Roberts WS, Fiorica JV. Detection of sentinel lymph nodes with lymphazurin in cervical, uterine, and vulvar malignancies. South Med J. 1999;92:204-208.

4. Frumovitz M, Ramirez PT, Levenback CF. Lymphatic mapping and sentinel lymph node detection in women with cervical cancer. Gynecol Oncol. 2008;110(3 suppl 2):S17-S20.

5. Oonk MH, Van de Nieuwenhof HP, De Hullu JA, Van der Zee AG. The role of sentinel node biopsy in gynecological cancer: a review. Curr Opin Oncol. 2009;21:425-432.

6. Van de Lande J, Torrenga B, Raijmakers PG, et al. Sentinel lymph node detection in early stage uterine cervix carcinoma: a systematic review. Gynecol Oncol. 2007;106: 604-613.

7. Abu-Rustum NR, Khoury-Collado F, Gemignani ML. Techniques of sentinel lymph node identification for early-stage cervical and uterine cancer. Gynecol Oncol. 2008;111:S44-S50.

8. Mais V, Peiretti M, Gargiulo T, Parodo G, Cirronis MG, Melis GB. Intraoperative sentinel lymph node detection by vital dye through laparoscopy or laparotomy in early endometrial cancer. J Surg Oncol. 2010;101:408-412.

9. Rossi EC, Ivanova A, Boggess JF. Robotically assisted fluorescence-guided lymph node mapping with ICG for gynecologic malignancies: a feasibility study. Gynecol Oncol. 2012;124:78-82.

10. Ferreira H, Nogueira-Silva C, Miranda A, Correia-Pinto J. Minilaparoscopy and sentinel lymph node in uterine cancer-preclinical study. J CRSLS. e2014.00055. doi:10.4293/CRSLS.2014.00055.

11. Occelli B, Narducci F, Lanvin D, et al. De novo adhesions with extraperitoneal endosurgical para-aortic lymphadenectomy versus transperitoneal laparoscopic para-aortic lymphadenectomy: a randomized experimental study. $A m$ J Obstet Gynecol. 2000;183:529-533.

12. Cohen J. Statistical Power Analysis for the Behavioral Sciences. 2nd ed. Hillsdale, NJ: Erlbaum; 1988.

13. Carlson RW, Allred DC, Anderson BO, et al. NCCN Clinical Practice Guidelines in Oncology, Breast Cancer version 2.2011. Fort Washington, PA: National Comprehensive Cancer Network; 2011.

14. Coit DG, Andtbacka R, Bichakjian CK, et al. Clinical Practice Guidelines in Oncology, Melanoma version 1.2011. Fort Washington, PA: National Comprehensive Cancer Network; 2011.

15. Van der Zee AG, Oonk MH, De Hullu JA, et al. Sentinel node dissection is safe in the treatment of early-stage vulvar cancer. J Clin Oncol. 2008;26:884-889.

16. Levenback C. Update on sentinel lymph node biopsy in gynecologic cancers. Gynecol Oncol. 2008;111(suppl 2):S42-S43.

17. Wishart GC, Loh SW, Jones L, Benson JR. A feasibility study (ICG-10) or indocyanine green (ICG) fluorescence 
mapping for sentinel lymph node detection in early breast cancer. Eur J Surg Oncol. 2012;38:651-656.

18. Sinno AK, Fader AN, Roche KL, Giuntoli RL, Tanner EJ. A comparison of colorimetric versus fluorometric sentinel lymph node mapping during robotic surgery for endometrial cancer. Gynecol Oncol. 2014;134:281-286.

19. Jewell E, Huang JJ, Abu-Rustum N, et al. Detection of sentinel lymph nodes in minimally invasive surgery using indocyanine green and near-infrared fluorescence imaging for uterine and cervical malignancies. Gynecol Oncol. 2014;133:274-277.

20. Dargent D, Salvat J. L'Envahissement ganglionnaire pelvien. New York, NY: McGraw Hill; 1989:150.

21. Querleu D, Dargent D, Ansquer Y, LeBlanc E, Narducci F. Extraperitoneal endosurgical aortic and common iliac dissection in the staging of bulky or advanced cervical carcinomas. Cancer. 2000;88:1883-1891.

22. Ramirez P, Milam M, Laparoscopic lymphadenectomy in patients with locally advanced cervical cancer. Gynecol Oncol. 2007;104(2 suppl 1):S9-S12.
23. Dargent D, Ansquer Y, Mathevet P. Technical development and results of left extraperitoneal laparoscopic paraaortic lymphadenectomy for cervical cancer. Gynecol Oncol. 2000;77:87-92.

24. Franco-Camps S, Cabrera S, Xercavins J, et al. Extraperitoneal laparoscopic approach for diagnosis and treatment of aortic lymph node recurrence in gynecologic malignancy. J Minim Invasive Gynecol. 2010;17:570-575.

25. Vasilev SA, Mcgonigle KF. Extraperitoneal laparoscopic para-aortic lymph node dissection. Gynecol Oncol. 1996;61:315-320.

26. Krpata DM, Ponsky TA. Needlescopic surgery: what's in the toolbox? Surg Endosc. 2013;27:1040-1044.

27. Bogani G, Uccella S, Cromi A, et al. Low vs standard pneumoperitoneum pressure during laparoscopic hysterectomy: a prospective randomized trial. J Minim Invasive Gynecol. 2013;21:466-471.

28. Zupi E, Marconi D, Sbracia M, et al. Is local anesthesia an affordable alternative to general anesthesia for minilaparoscopy? J Am Assoc Gynecol Laparosc. 2000;7:111-114. 